Lengua y Sociedad, revista de lingüística teórica y aplicada

Vol. 17, n. ${ }^{\circ} 2$, Lima, julio-diciembre de 2018, pp. 45-61

https://doi.org/10.15381/lengsoc.v17i2.22352

\title{
Superando el principio de equivalencia semántica: un caso de variación morfológica estudiado con el concepto de espacios de variabilidad $^{1}$
}

\section{Overcoming the requirement of semantic equivalence: a case of morphological variation studied with variability's spaces concept}

\author{
Anahís Samamé Rispa \\ Pontificia Universidad Católica del Perú \\ anahis.samamer@pucp.edu.pe
}

\begin{abstract}
Resumen
Desde la sociolingüística variacionista, se estudia un caso de variación morfológica, el uso de las formas de imperfecto y pluscuamperfecto de subjuntivo del español (-ra/-se), a través del concepto de espacios de variabilidad postulado por Caravedo (1991, 2014), el cual supera el requisito de equivalencia semántica que plantea la variable sociolingüística. A partir de una muestra de 54 castellanohablantes nacidos en Lima con formación universitaria, se investiga el fenómeno con el fin de determinar si la variante -ra está desplazando a -se. De la misma manera, se pretende conocer si la elección de una u otra forma está condicionada estadísticamente por la variable lingüística tiempo verbal. Se concluye que el uso de -ra supera al de -se en la muestra estudiada. Asimismo, el tiempo verbal se mostró como una variable significativa estadísticamente: el tiempo verbal simple se relaciona con el uso de -ra y el compuesto, con mayores usos de -se.
\end{abstract}

Palabras clave: tiempo verbal, variación morfológica, variable, castellano, Lima, espacios de variabilidad.

\begin{abstract}
From the field of sociolinguistics, this research studies a case of morphological variation, the use of imperfect and pluperfect subjunctive of Spanish (-ra/-se), through the concept of variability's spaces postulated by Caravedo $(1991,2014)$, wich overcames the sociolinguistic variable's requirement of semantic equivalence. The sample is formed by 54 Spanish speakers born in Lima with university education. The phenomenon is investigated in order to determine if the variant -ra is displacing the variant -se. In the same way, it is intended to know if the choice of one form or another is statistically conditioned by the verbal tense. It is concluded that the use of -ra exceeds the use of -se in the sample studied. Likewise, verbal tense was shown as a statistically significant variable: simple tense is related to the use of $-r a$ and compound tense, with -se.
\end{abstract}

Keywords: verbal tense, morphological variation, variable, Spanish, Lima, spaces of variability.

1 Este artículo presenta parte de los resultados de mi trabajo de tesis de maestría, el cual fue financiado por el Programa de Apoyo a la Investigación para Estudiantes de Posgrado (PAIP) 2018 de la PUCP. Se agradecen los comentarios brindados por los profesores de la Maestría y, en especial, los de mi asesor de tesis, Jorge Iván Pérez. Cualquier error recae sobre mi responsabilidad. 


\section{Anahís Samamé Rispa}

\section{Introducción}

Desde los primeros estudios de Labov en la década de 1960, los cuales dieron inicio al nacimiento de la sociolingüística variacionista, ha habido una amplia discusión acerca del concepto de variable y sus límites en cuanto a su aplicación a otros planos más allá del fonológico (Lavandera, 1984 [1978 y 1979]; Labov, 1978; Caravedo, 1987). La discusión ha girado en torno al principio de equivalencia semántica que el autor postula como condición para determinar cuándo se está ante una variable. Este principio resulta problemático si se aplica a variaciones de tipo morfológico o sintáctico, por ejemplo, ya que las formas que varían difícilmente serán completamente equivalentes al contar cada una con significados distintos, al contrario de lo que sucede con casos de variación fonológica.

En este trabajo, desde el campo de la sociolingüística variacionista, se estudia un caso de variación morfológica, el uso de las formas de imperfecto y pluscuamperfecto de subjuntivo del español (-ra/-se), a través del concepto de espacios de variabilidad postulado por Caravedo (1991, 2014). A partir de una muestra de 54 hablantes de español nacidos en Lima y que cuentan con formación universitaria, se investiga el fenómeno con el fin de determinar si la variante - ra está desplazando a la variante -se en la muestra estudiada. De la misma manera, se pretende conocer si la elección de una u otra forma está condicionada estadísticamente por la variable de corte lingüístico tiempo verbal, el cual se entiende como simple (cantara o cantase) o compuesto (hubiera cantado o hubiese cantado) para fines de esta investigación. Con lo anterior, se busca demostrar la funcionalidad del concepto de espacios de variabilidad, el cual deja de lado el requisito de equivalencia semántica, para estudiar casos de variación en los que el significado está implicado, como el del presente trabajo.

En la siguiente sección, se introduce la problemática que plantea el concepto de variable para los estudios más allá del plano fonológico y se brinda un panorama sucinto del estado de este fenómeno de variación a través de la revisión de algunos estudios sobre el tema en España, Venezuela y Perú. Luego, se explica la metodología seguida para la obtención de los datos: se específica la muestra de hablantes y el tipo de cuestionario utilizado para elicitar las formas de interés. En la cuarta sección, se presenta el análisis de los datos y los resultados obtenidos. Finalmente, se ofrecen las conclusiones a las que el análisis permite llegar. 
Superando el principio de equivalencia semántica: un caso de variación...

\section{Estado del arte}

En esta sección, se plantea la problemática que representa el principio de equivalencia semántica como requisito de la variable lingüística para los estudios en los que el significado está involucrado. Además, se ofrece un panorama general de las investigaciones llevadas a cabo con el fin de conocer el estado de la variable en cuestión, tanto acerca de las frecuencias de uso de -ra y -se, así como de su condicionamiento por la variable lingüística de tiempo verbal.

\subsection{El principio de equivalencia semántica como requisito de la variable lingǘística ${ }^{2}$}

En 19783, Lavandera escribe un artículo en el que reflexiona sobre los límites de la aplicación del concepto de variable sociolingüística, entendida como una unidad con manifestaciones diversas, al plano sintáctico, y problematiza el principio de equivalencia semántica propuesto como requisito de esta por Labov (1983 [1972]). La crítica va dirigida al hecho de que, en el plano sintáctico, las formas cuentan con un significado referencial del que están desprovistas las variables fonológicas, por lo que la tarea de demostrar que dos enunciados con distinta forma tienen el mismo significado referencial se complejiza. De esta manera, para la autora, el concepto de variable (socio) lingüística se presenta como insuficiente o inadecuado para aplicarlo al estudio de la variación más allá de la fonológica, como la variación sintáctica.

Para superar la problemática anterior, Lavandera (1984 [1978]) postula el concepto de comparabilidad funcional como sustituto del requisito de equivalencia semántica de la variable, aunque no lo desarrolla. A partir del estudio semántico de las prótasis en construcciones condicionales de la autora (Lavandera, 1984 [1979]), Caravedo (1987) ofrece una interpretación de este nuevo concepto. Así, menciona que, aunque las formas verbales (presente de indicativo canto, condicional cantaría e imperfecto de subjuntivo cantara/cantase) sincrónicamente se usan en contextos diferentes y con funciones diferenciadas, se pueden estudiar

2 Para una versión más extendida de la discusión que aquí se presenta, puede verse el trabajo de tesis del que parte este escrito (Samamé 2018, pp. 37-42). Las investigaciones a las que se hace referencia corresponden a Lavandera (1984 [1978 y 1979]), Labov (1978) y Caravedo (1987, 1991, 2014).

3 Presenta una reimpresión de este artículo en su libro Variación y significado (Lavandera, 1984), texto que fue revisado para este trabajo. 
como variantes de una misma variable, porque «[...] en el plano sintáctico pueden coaparecer en un tipo de estructura como la condicional» (Caravedo, 1987, p. 211). Aun cuando la interpretación que ofrece la investigadora parece bastante acertada, no se termina de delimitar cuáles serían esos elementos que podrían «coaparecer» en las condicionales: no se especifica a qué clase de elementos se hace referencia ni el lugar que ocuparán en la estructura sintáctica.

Por lo explicado anteriormente, se sostiene que el concepto de comparabilidad funcional no terminaría de dar cuenta de forma precisa del caso de variación estudiado en la presente investigación. Por ello, se propone trabajar con la propuesta de espacios de variabilidad de Caravedo (1991, 2014): «[...] un conjunto de posibilidades que he denominado espacio de variabilidad (Caravedo, 1990, 1991). Se trata de un continuum sonoro dentro del cual se hacen posibles ciertas fluctuaciones, que tienen determinados límites [...] [que] pueden o no coincidir con la capacidad discriminativa de significado» (Caravedo, 2014, pp. 130-131). Aunque la propuesta nace pensando en variación fonológica (1991), en 2014, la autora la aplica al plano sintáctico y léxico, y la amplía al plano conceptual: «[...] sostendré que la variación [...] puede ondular entre zonas conceptuales distintas, las cuales se acercan y se intersectan en determinados contextos» (Caravedo, 2014, p. 159). De esta manera, «[...] podrá existir una variación en el plano del significante léxico o sintáctico [...] o una variación en el plano del significado mismo atribuido a una forma material única» (Caravedo 2014, p. 158). Así, claramente, ya queda superado el principio de equivalencia semántica: se trata de conceptos, por ende, diferentes, que «comparten» o se «intersectan» en ciertos espacios y se actualizan en una misma forma.

Entonces, el criterio por el que se decide estudiar a las formas - ra y -se como variantes de un caso de variación morfológica en este trabajo radica justamente en el concepto de espacios de variabilidad. Para esto, se propone extender su aplicación más allá de los casos estudiados por la autora para el plano sintáctico y léxico (Caravedo, 2014), en donde la variación ocurre en el plano conceptual pero no del significante. Como ya se sabe, el objetivo del presente trabajo es estudiar un caso de variación morfológica de dos formas diferentes que coaparecen en diversos contextos (-ra y -se) y que presentan, además, diferencias semánticas - como se demostrará-. Así, lo que justificaría agrupar a las formas en cuestión como parte de un mismo fenómeno de variación es que - ra y -se compartirían un mismo espacio de variabilidad. En otras palabras, se encuentran en un 
Superando el principio de equivalencia semántica: un caso de variación...

mismo continuum que posibilita su coaparición y cuyos límites se muestran en este estudio con el análisis respectivo.

De la discusión de arriba, se desprende el hecho de que este nuevo concepto de Caravedo es compatible con el de comparabilidad funcional de Lavandera. Aunque lo que sigue no es afirmado por ninguna de las autoras, el primer concepto recoge al segundo en cierto sentido, ya que las variantes que comparten un espacio de variabilidad aparecen en un determinado contexto, lo que las haría «comparables funcionalmente». De esta manera, el concepto de espacios de variabilidad permite explicar de forma más precisa y adecuada la variación entre -ra y -se. Además, este concepto es más abarcador, ya que permite estudiar la variación a nivel léxico y no solo a nivel morfosintáctico o sintáctico.

\subsection{Antecedentes}

En cuanto a las frecuencias de uso de las formas de imperfecto y pluscuamperfecto de subjuntivo -ra y -se, diversos estudios se han llevado a cabo en distintas ciudades de España y Latinoamérica para dar cuenta del uso mayoritario de la primera en detrimento de la segunda. Autores como Wright (1926), Williams (1982) y DeMello (1993) se centran en estudiar este fenómeno de variación en Latinoamérica, España y ambos espacios, respectivamente. Los resultados obtenidos confirman el uso mayoritario de - ra sobre -se, afirmación bastante generalizada en los estudios al respecto, como señalan los mismos autores y como se seguirá sustentando más adelante con aquellos trabajos que abarcan el fenómeno desde una perspectiva variacionista.

Para el caso de Perú, solo se han encontrado dos investigaciones. Una de estas pertenece al autor citado anteriormente, DeMello (1993), quien es el único que trabaja con datos de la variedad limeña. Este investigador estudia el fenómeno mediante entrevistas a hablantes de español culto de diez ciudades de América y España ${ }^{4}$. Los datos obtenidos para la ciudad de Lima muestran el porcentaje más bajo (o.6 \%) para las formas en -se en comparación con las otras ciudades. El otro estudio del contexto peruano pertenece a Cuba (1996), quien trabaja con datos de la variedad del castellano de Chincha. El patrón de distribución de las variantes -ra y-se que encuentra la autora es opuesto al descrito anteriormente; es

4 Bogotá, Buenos Aires, Caracas, La Habana, Lima, Madrid, Ciudad de México, San Juan de Puerto Rico, Santiago de Chile y Sevilla 
decir, para el castellano chinchano, se evidenció que la tendencia sería un mayor uso de hubiese que de hubiera (1996, p. 34). Lo anterior resulta bastante interesante y sería necesario una indagación más profunda para corroborar los datos y conocer las posibles causas por que el uso de las formas muestra una tendencia opuesta a la general.

Respecto a las investigaciones de corte variacionista que incluyen la variable de tiempo verbal (simple o compuesto) como factor lingüístico involucrado en la elección de una u otra forma, en España, Blas y Porcar (1992) estudian el fenómeno en comunidades castellonenses de habla castellana y valenciana. Los autores encuentran que, además de las frecuencias de uso más altas de -ra que de -se, existe una tendencia a la selección de -se en formas compuestas del verbo en oraciones subordinadas adverbiales concesivas y condicionales, sobre todo, en las primeras. A pesar de obtener este resultado, los investigadores no concluyen de forma definitiva acerca de los matices semánticos distintos que revestirán a las variantes.

Por su parte, Serrano (1996) estudia la distribución de las variantes del imperfecto de subjuntivo en oraciones condicionales potenciales e irreales del español de La Laguna (Tenerife) y demuestra que esta no es aleatoria. Aunque la autora no incluye en su estudio la variable de tiempo verbal simple o compuesto, las conclusiones a las que llega son importantes para lo que pretende demostrar en el presente trabajo, como se podrá observar más adelante. Si bien Serrano encontró el predominio de - $r a$ sobre -se en ambos tipos de condicionales (70 \% y $30 \%$ respectivamente para potenciales, y $60 \%$ y $40 \%$ respectivamente para irreales), se observó un mayor uso de -se en irreales y de -ra en potenciales motivado por los usos históricos de ambas formas: la primera forma proviene del pluscuamperfecto de subjuntivo del latín, mientras que la segunda, del pluscuamperfecto de indicativo. Además, en ambas, la aparición de -ra se vio favorecida por la presencia de indicativo en la apódosis, mientras que -se tiene más apariciones cuando se usa un condicional en la apódosis.

En Venezuela, son dos los estudios que se han llevado a cabo sobre esta variable. Navarro (1990) investiga el imperfecto y pluscuamperfecto de subjuntivo en la ciudad de Valencia para conocer la frecuencia de uso de -se frente a - $r a$ en contextos donde son sintácticamente equivalentes, y - ra frente a -ría en la apódosis de las condicionales, así como para averiguar si su índice de frecuencia en el español valenciano se halla condicionado por factores lingüísticos y/o sociales. El autor encontró que las formas en -se solo alcanzaron el $9 \%$ del total de producciones, con lo que comprueba el uso mayoritario de -ra en Valencia.

50 Lengua y Sociedad 
Superando el principio de equivalencia semántica: un caso de variación...

En cuanto a la variable tiempo, mientras que solo se obtuvo un índice de $5 \%$ para -se en oraciones de tiempo simple, su porcentaje aumentó a un $37 \%$ en cláusulas con tiempo compuesto. Otro factor lingüístico que sería importante mencionar en este estudio es el de contexto de prótesis, ya que este aumentaría el uso de la forma en $-s e$.

Asratián (2007) estudia el uso de las formas en la variedad de Caracas. Como los demás autores citados, se demuestra en uso mayoritario de -ra (94\%) en comparación con -se (6\%) para el habla caraqueña. Una de las variables lingüísticas que la autora encuentra como significativa estadísticamente es el tiempo compuesto, el cual favorecería el empleo de -se, a diferencia del tiempo simple. La negación fue otra de las variables que se mostró como un factor de influencia para una mayor aparición de -se en el trabajo de la autora.

Aunque no existe consenso entre los autores que han estudiado el fenómeno y algunos de ellos sostengan que las formas de imperfecto y pluscuamperfecto de subjuntivo se encuentran en libre alternancia y no implican diferencias semánticas (Alarcos Llorach, 1949, citado en Lamíquiz, 1971, p. 2; Alvar y Pottier, 1983; RAE, 2009), los estudios a los que se ha hecho referencia arriba demuestran que, aun cuando el uso mayoritario de -ra está extendido en casi todas las variedades de habla española, la variable de tiempo verbal compuesto se comporta como un factor lingüístico importante para el aumento de las frecuencia de uso -se en los contextos en que esta aparece. Lo anterior se puede explicar a partir de la información etimológica de las formas con las diferencias semánticas que esta implica. Es esta afirmación la que se intenta demostrar en este artículo.

\section{Metodología}

\subsection{1. Población y muestra}

La población objetivo está conformada por todos los hablantes nativos de español nacidos en la ciudad de Lima que se encuentren cursando o que hayan cursado estudios universitarios. La muestra de esta investigación consiste en 54 sujetos divididos por género ( 27 mujeres y 27 hombres) y edad (jóvenes, adultos y mayores). La distribución por rangos de edad es como sigue: 18 hablantes jóvenes de 20 a 34 años (9 mujeres y 9 hombres), 18 adultos de 35 a 50 años ( 9 mujeres y 9 hombres) 
y 18 colaboradores mayores de 51 a más años de edad (9 mujeres y 9 hombres). Todos los entrevistados cumplen con las características de haber nacido en el departamento de Lima, tener al castellano como lengua materna y cursar o haber cursado estudios superiores universitarios.

\subsection{Instrumento de recolección de datos}

El instrumento de recolección de datos (IRD) consta de un cuestionario anónimo que contiene cuatro partes. Cada parte del cuestionario buscaba elicitar datos específicos. La primera, por ejemplo, recogía los datos personales del colaborador para clasificar al hablante por edad y género. En la segunda parte, a partir de imágenes de diferentes escenas de un video que habían visto previamente, se les pedía a los encuestados que imaginen situaciones distintas o contrarias a las que observaron y completen con formas verbales los espacios en blanco de las preguntas propuestas, así como de las respuestas a esas preguntas. En este segundo apartado del IRD, hubo un total de 20 oraciones condicionales (10 hacen referencia a las preguntas y 10, a las respuestas). La tercera parte del cuestionario incluía cinco imágenes acompañadas de una breve historia con 8 oraciones con espacios en blanco para ser completados con las formas verbales de interés. Para esto, se les pedía a los colaboradores imaginar lo que los personajes de las imágenes podrían estar diciendo o pensando. La última parte del cuestionario fue un texto para completar adaptado del estudio de Williams (1982) para el español de Navarra, España. En este apartado, se incluyen 13 oraciones en las que necesariamente deberían aparecer las formas en subjuntivo y 7 oraciones distractoras para completar con otras formas verbales.

\section{Resultados}

En este apartado, se presentan los resultados del análisis estadístico llevado a cabo para la variable en cuestión. Las dos siguientes subsecciones ofrecen el análisis descriptivo: se trata del conteo total de las formas elicitadas - ra y -se, y del conteo de estas para la variable de tiempo verbal, respectivamente. La tercera subsección incluye el análisis correlacional con el fin de conocer si existe asociación estadística entre el imperfecto y pluscuamperfecto de subjuntivo, y el tiempo verbal. 
Superando el principio de equivalencia semántica: un caso de variación...

\subsection{Conteo total de datos ${ }^{5}$}

La tabla 1 muestra las frecuencias absolutas obtenidas para el total de las formas -ra y -se, tanto de imperfecto como de pluscuamperfecto de subjuntivo. Además, ofrece los porcentajes de cada una de estas, los cuales se pueden visualizar de manera más gráfica más abajo.

Tabla 1. Conteo total de las formas en -ra y-se

\begin{tabular}{l|l|l}
\cline { 2 - 3 } & Frecuencia & Porcentaje \\
\hline ra & 1266 & 79.6 \\
\hline se & 324 & 20.4 \\
\hline Total & 1590 & 100.0 \\
\hline
\end{tabular}

\section{Gráfico 1. Conteo total de las formas en -ra y-se}



\footnotetext{
5 El total de formas recolectadas en la muestra estudiada a través del IRD fue de 2214. De estas, solo 1590 correspondían a las formas de imperfecto y pluscuamperfecto de subjuntivo y el resto, a formas del presente de subjuntivo, condicional, indicativo y otros. En el trabajo de tesis del que parte este artículo, se ofrecen, de manera detallada, las frecuencias y porcentajes obtenidos para cada uno de los elementos verbales recolectados. Por razones de espacio, en el presente artículo, solo se consideran las variantes de -ra y -se para el análisis.
} 
Como se puede observar en la tabla y gráfico 1, existe una tendencia bastante clara al mayor uso de -ra en detrimento de -se. Así, de las 1590 formas obtenidas para el imperfecto y pluscuamperfecto de subjuntivo en este estudio, 1266 pertenecen a -ra, es decir, casi el $80 \%$. Por su parte, las formas en -se solo ocupan la cuarta parte de la variante anterior $(20,4 \%)$ con 324 apariciones del total.

El conteo anterior permite afirmar que el castellano limeño cumple el mismo patrón de uso de las formas - ra y -se que las demás variedades del castellano estudiadas en cuanto a este aspecto. Así, se comprueba la tendencia a un escaso uso de -se en los hablantes de Lima, lo que se corresponde con lo que sucede en otras ciudades tanto de Latinoamérica como de España.

\subsection{Conteos para la variable tiempo verbal}

A continuación, se presentan los resultados de los conteos obtenidos para la variable de tiempo verbal: primero, el tiempo simple y, luego, el tiempo compuesto. Como se podrá observar, en este caso, se detallan las apariciones de las formas de imperfecto y las de pluscuamperfecto para cada variante.

\subsubsection{Conteos para el tiempo verbal simple}

Tabla 2. Conteos del tiempo simple

\begin{tabular}{l|l|l}
\cline { 2 - 3 } \multicolumn{1}{c|}{} & \multicolumn{1}{c|}{ Frecuencia } & \multicolumn{1}{c}{ Porcentaje } \\
\hline ra imperfecto & 631 & 80.5 \\
\hline se imperfecto & 98 & 12.5 \\
\hline ra pluscuamperfecto & 29 & 3.7 \\
\hline se pluscuamperfecto & 26 & 3.3 \\
\hline Total & 784 & 100.0 \\
\hline
\end{tabular}

Según los datos proporcionados en la tabla 2, de los contextos que buscaban evaluar el tiempo verbal simple en la data, se obtuvieron 784 formas de imperfecto y pluscuamperfecto de subjuntivo. Como es de esperarse, la mayor cantidad de apariciones corresponden al imperfecto: 631 veces apareció - $r a(80,5 \%)$ y 98 veces, $-s e(12,5 \%)$. Es decir, en este caso, el porcentaje de esta última variante baja a poco más de la sexta parte de -ra. Además, los hablantes produjeron formas 
Superando el principio de equivalencia semántica: un caso de variación...

de pluscuamperfecto mínimamente: estas solo aparecen 55 veces, de las que 29 corresponden a -ra $(3,7 \%)$ y 26 , a $-s e(3,3 \%)$.

Es interesante notar dos aspectos. En primer lugar, en los contextos de tiempo verbal simple, las producciones de -ra superan en más de seis veces a las -se. Aunque, en general, se espera una frecuencia de uso de - $r a$ alta, en este contexto específico, parece ser que la tendencia a la aparición de esta forma es aun mayor. En segundo lugar, según los porcentajes del pluscuamperfecto, -ra no supera por mucho a -se, como sí ocurre con el imperfecto, ya que la primera forma solo ocurre 0,4\% más que la segunda; es decir, la diferencia es mínima. Estos datos son reveladores en el sentido de que, con las formas de pluscuamperfecto (tiempo compuesto), no se parece cumplir el patrón de distribución general del uso mayoritario de -ra sobre -se.

\subsubsection{Conteos para el tiempo verbal compuesto}

Tabla 3. Conteos del tiempo compuesto

\begin{tabular}{l|l|l}
\cline { 2 - 3 } & \multicolumn{1}{c|}{ Frecuencia } & \multicolumn{1}{c}{ Porcentaje } \\
\hline ra imperfecto & 15 & 1.9 \\
\hline se imperfecto & 4 & .5 \\
\hline ra pluscuamperfecto & 591 & 73.3 \\
\hline se pluscuamperfecto & 196 & 24.3 \\
\hline Total & 806 & 100.0
\end{tabular}

Como se puede observar en la tabla 3, en las cláusulas mediante las que se pretendía evaluar la variable de tiempo verbal compuesto, se produjeron 806 veces las formas de subjuntivo esperadas: 606 apariciones de -ra y 200 apariciones de -se. De estas, solo 19 corresponden al imperfecto ( 15 veces - $r a$ y 4 veces $-s e$ ), mientras que 787 , al pluscuamperfecto. Sobre las últimas, 591 producciones son de -ra y 196, de -se: en este caso, el porcentaje de la primera variante solo supera a la segunda por poco más del triple que esta.

Los datos anteriores permiten evidenciar diferencias importantes con respecto a la aparición de las formas tomando en cuenta la variable de tiempo verbal. Así, parece ser que, aunque la frecuencia de las formas en -ra siempre es mayoritaria, la proporción de uso varía de forma resaltante: en el contexto de tiempo simple, 
esta variante aparece más del séxtuple de veces que -se; en cambio, en el tiempo compuesto, solo se produce más del triple. Esta diferencia se puede apreciar de forma más clara en el gráfico 2, en el que se comparan las frecuencias relativas de uso de las variantes de forma más simplificada atendiendo solo a la diferencia de tiempo verbal.

\section{Gráfico 2. Comparación del conteo de la variable tiempo verbal}

a) Tiempo simple

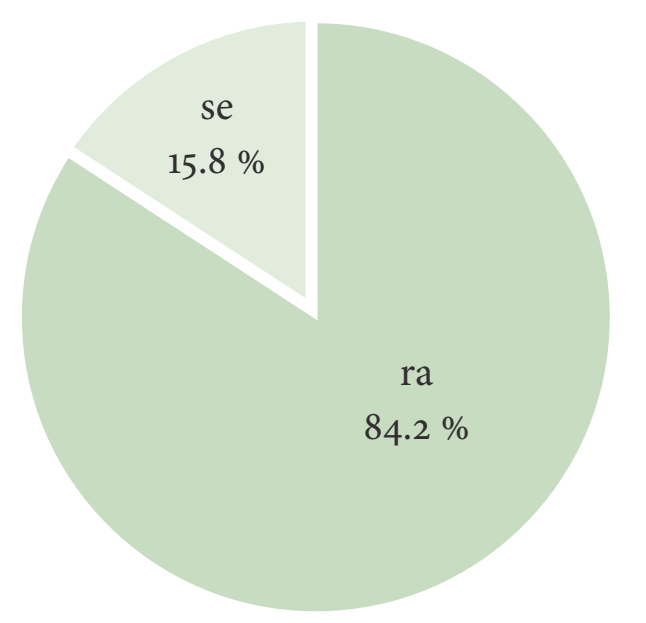

b) Tiempo compuesto

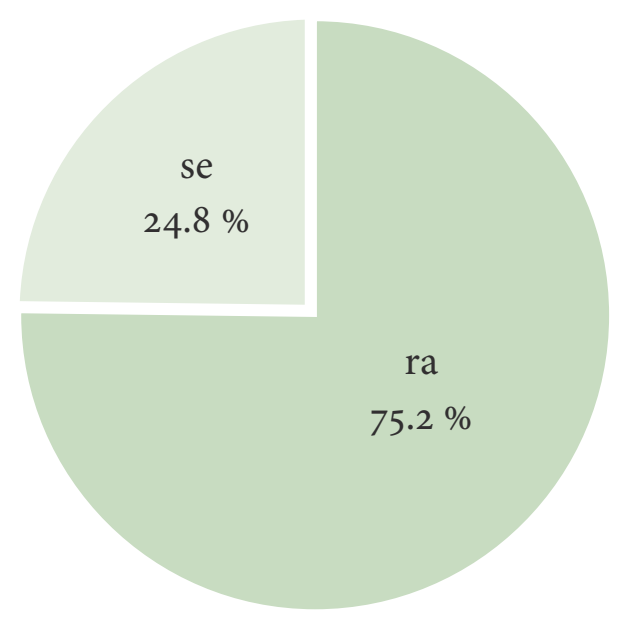

El gráfico 2 permite observar de forma más clara la diferencia en cuanto al porcentaje de apariciones de las formas - $r a$ y -se a partir de la variable tiempo verbal. En el tiempo simple (gráfico 2a), -ra aparece el 84,2 \% del total y -se, el $15,8 \%$. En cambio, el porcentaje de -ra disminuye a 75,2 \% y el de -se sube a $24,8 \%$ en el tiempo compuesto (gráfico 2b). Aunque los valores del uso de -ra son por mucho más altos a los de -se en ambos contextos, no es gratuito que los valores de -se aumenten en $9 \%$ más en el tiempo compuesto que en el simple. Se sostiene, por tanto, que es el primer contexto el que favorece la presencia de las formas en -se, a diferencia de lo que sucede con el tiempo verbal simple.

\subsection{Correlación con la variable tiempo verbal}

En esta sección, se ofrecen los resultados obtenidos a partir del análisis estadístico correlacional con el programa spss aplicado con el fin de conocer si existe 
Superando el principio de equivalencia semántica: un caso de variación...

correlación entre la variable dependiente, imperfecto y pluscuamperfecto de subjuntivo, y la variable lingüística independiente, tiempo verbal. Para lo anterior, primero, se aplicó la prueba de Chi-cuadrado. Luego, para entender el tipo de asociación entre las variables, la técnica de correspondencia simple resultó útil. A continuación, se presentan las tablas que dan cuenta de lo anterior.

Tabla 4. Tabulación cruzada: tiempo verbal y variable dependiente

\begin{tabular}{|c|c|c|c|c|}
\hline & & \multirow{2}{*}{\multicolumn{2}{|c|}{ Tiempo }} & \multirow{3}{*}{ Total } \\
\hline & & & & \\
\hline & & Simple & Compuesto & \\
\hline \multirow[t]{2}{*}{ Formas } & $\mathrm{Ra}$ & 631 & 591 & 1222 \\
\hline & $\mathrm{Se}$ & 98 & 196 & 294 \\
\hline Total & & 729 & 787 & 1516 \\
\hline
\end{tabular}

El conteo de las formas - ra y -se para cada tiempo verbal se muestra en la tabla 4. Para conocer si existe asociación estadística entre las variables, se manejan dos hipótesis, a las que se aplicó la técnica de Chi-cuadrado con el fin de probarlas.

$\mathrm{H}_{\mathrm{o}}$ : Las formas de imperfecto y pluscuamperfecto de subjuntivo, y el tiempo verbal no se relacionan.

$\mathrm{H}_{\mathrm{i}}$ : Las formas de imperfecto y pluscuamperfecto de subjuntivo, y el tiempo verbal sí se relacionan.

Tabla 5. Prueba de Chi-cuadrado para tiempo verbal

\begin{tabular}{|c|c|c|c|}
\hline & Valor & gl & $\begin{array}{l}\text { Sig. asintótica } \\
\text { ( } 2 \text { caras })\end{array}$ \\
\hline Chi-cuadrado de Pearson & $31,804^{\mathrm{a}}$ & 1 &, 000 \\
\hline Razón de verosimilitud & 32,390 & 1 &, 000 \\
\hline $\mathrm{N}$ de casos válidos & 1516 & & \\
\hline
\end{tabular}

Nivel de significación: 0.01

El valor del Sig. asintótica de la tabla 5 es menor al nivel de significación $(0,00<0,01)$, por lo que se rechaza la hipótesis nula $\left(\mathrm{H}_{\mathrm{o}}\right)$ y se confirma la hipótesis de investigación $\left(\mathrm{H}_{\mathrm{i}}\right)$. Por lo anterior, se logra concluir, con un nivel de significación del $1 \%$, que las formas de imperfecto y pluscuamperfecto, y el tiempo 
verbal están asociados estadísticamente, lo que comprueba la hipótesis de esta investigación. Para saber cuál es la relación específica entre - ra y -se, se aplica la prueba de correspondencia simple a esta variable lingüística.

Tabla 6. Prueba de correspondencia simple para tiempo verbal y la variable dependiente

\begin{tabular}{|c|c|c|c|c|c|c|c|}
\hline \multirow{3}{*}{ Formas } & \multirow{3}{*}{ Masa } & \multirow{2}{*}{\multicolumn{2}{|c|}{$\begin{array}{l}\text { Puntuación } \\
\text { en dimensión }\end{array}$}} & \multirow{3}{*}{ Inercia } & \multicolumn{3}{|c|}{ Contribución } \\
\hline & & & & & \multirow{2}{*}{$\begin{array}{l}\text { Del punto en } \\
\text { la inercia de } \\
\text { dimensión } \\
1\end{array}$} & \multicolumn{2}{|c|}{$\begin{array}{l}\text { De la dimensión en la } \\
\text { inercia del punto }\end{array}$} \\
\hline & & & 1 & & & 1 & Total \\
\hline $\mathrm{Ra}$ & ,806 &,- 187 & &, 004 & 194 & 1,000 & 1,000 \\
\hline $\mathrm{Se}$ & 194 &, 776 & & ,017 & ,806 & 1,000 & 1,000 \\
\hline Total activo & 1,000 & & & ,021 & 1,000 & & \\
\hline
\end{tabular}

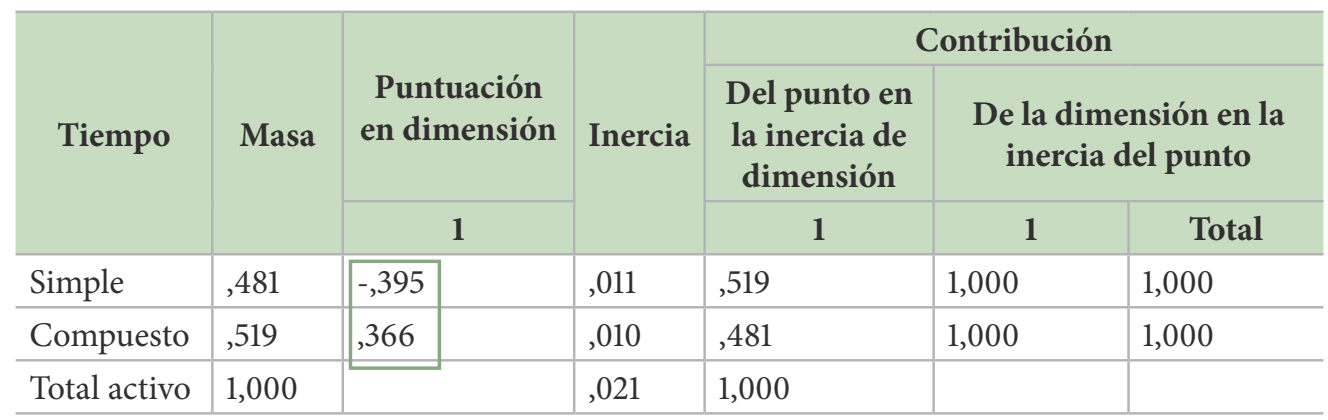

En la tabla 6, se puede observar que la puntuación para - ra tiene signo negativo, mientras que para -se, positivo. Por su parte, en cuanto al tiempo verbal, el tiempo simple tiene signo negativo y el tiempo compuesto, positivo. Por lo tanto, se puede concluir, estadísticamente, que la variante - $r a$ se asocia con las formas verbales simples y-se, con verbos compuestos, lo que comprueba el tipo de relación que se plantea en la hipótesis mencionada arriba.

Los datos anteriores corroboran un resultado obtenido en las investigaciones revisadas: la asociación entre el tiempo compuesto y la variante -se (Navarro, 1990; Asratián, 2007), lo cual se cumple en distintas variedades del español. De esta manera, el hecho de que sea el tiempo compuesto el contexto que genera una mayor aparición de las formas en -se no se restringe al castellano limeño solamente, sino que parece ser un rasgo general del español. La explicación que 
Superando el principio de equivalencia semántica: un caso de variación...

se postula se relaciona con las etimologías de las formas: -se proviene del pluscuamperfecto de subjuntivo del latín, mientras que - $r a$, del pluscuamperfecto de indicativo. Aunque ambas formas pertenecían al pluscuamperfecto, se diferenciaban en el modo: la primera forma pertenecía al modo que expresa no-realidad, deseo, duda, y la segunda pertenecía al modo asociado con significados más reales y concretos. Son estos significados los que, de alguna manera, parecen seguir vigentes en ambas formas.

Según Fleischman, la distancia temporal expresada a través de la categoría tiempo está sistemáticamente extendida para expresar distancia en otros dominios, como el pragmático, por ejemplo (1989:3). Ya que el tiempo compuesto está más alejado del presente (de lo actual) en comparación con el simple, se puede sostener que el primero está más asociado a lo [-real] que el segundo, así se trate de un pretérito en ambos casos. En ese sentido, la forma -se - que proviene del pluscuamperfecto del latín, como ya se mencionó-, es más compatible con las formas compuestas que con las simples. Parece ser que los valores menos reales con los que se asocian las formas de subjuntivo no han impregnado del todo a - ra, por lo que se retiene el uso de la forma -se en el contexto de tiempo compuesto al estar más asociada con su valor etimológico aún.

Como se ha demostrado, las formas - ra y -se del imperfecto y pluscuamperfecto de subjuntivo poseen matices semánticos diferentes $y$, aun así, pueden coaparecer en determinados contextos. El concepto de variable sociolingüística se plantea problemático para estudiar este caso de variación morfológica teniendo en cuenta que se debe respetar el principio de equivalencia semántica, según el cual las variantes no deben representar diferencias de significado. De esta manera, la propuesta de espacios de variabilidad de Caravedo resulta útil en este estudio. Se observa cómo las variantes analizadas fluctúan en un mismo espacio, en un mismo continuum, lo que permite estudiarlas como un caso de variación sociolingüística sin la necesidad de entenderlas como dos elementos que se desprenden de una misma unidad en la que no habría diferencias de tipo semántico.

\section{Conclusiones}

En esta investigación, las frecuencias obtenidas para las formas en cuestión fueron de 79, $6 \%$ para - ra y 20, $4 \%$ para $-s e$. En ese sentido, para la variedad del castellano limeño, se comprueba el uso mayoritario de - ra sobre -se, como sucede 
en la mayor parte de investigaciones realizadas sobre este fenómeno. Se puede sostener, así, que la preferencia de la primera variante por la segunda es general al habla castellana, salvo algunas excepciones como la variedad del español hablado en Chincha.

En cuanto a la variable lingüística de tiempo verbal, las apariciones de -ra aumentan en $9 \%$ más en el tiempo simple que en el compuesto, mientras que con las de -se sucede lo contrario: aumentan en $9 \%$ más pero en el tiempo compuesto en comparación con el simple. A estos datos se les aplicó el análisis mediante el programa sPss, el cual arrojó asociación estadística entre el tiempo verbal, y la variable de imperfecto y pluscuamperfecto de subjuntivo. Mientras que las formas en - $r a$ se relacionaban estadísticamente con el tiempo simple, las formas en -se, con el tiempo compuesto.

Esta asociación encuentra una explicación en la etimología de las formas: la procedencia de - ra del pluscuamperfecto del indicativo del latín, modo de la realidad, de lo actual, de lo presente, y de -se del pluscuamperfecto del subjuntivo, modo de la no-realidad, del deseo, de la duda. Estas diferencias parecen seguir vigentes en el castellano actual. Aunque tanto el tiempo simple como el compuesto pertenecen al pasado, se prefiere las formas en -se con este último, porque se encuentra más alejado del presente en comparación con el simple, contexto en el que se selecciona una frecuencia más alta de - ra. A partir de lo anterior, se sostiene que el estudio de este fenómeno a través del concepto de espacios de variabilidad resulta funcional, ya que este deja de lado el requisito de equivalencia semántica de variable sociolingüística para estudiar casos de variación en los que se encuentran matices diferentes de significado en las formas implicadas, como sucede en el presente trabajo.

\section{Bibliografía}

Alvar, M., \& Pottier, B. (1983). Morfología histórica del español. Madrid: Gredos. Asratián, A. (2007). Variación -ra/-se en el español hablado en Caracas. Boletín de Lingüística, 19(27), 5-41. Recuperado de http://www.scielo.org.ve/scielo. php?pid=So798-97092007000100001\&script=sci_arttext.

Blas, J., \& Porcar, M. (1994). Empleo de las formas -ra y -se en las comunidades de habla castellonenses. Aproximación sociolingüística. Español actual. Revista de español vivo, 62, 73-98. 
Superando el principio de equivalencia semántica: un caso de variación...

Caravedo, R. (1987). Reseña de Variación y significado. Filología, 22(1), 207-212.

Caravedo, R. (1991). Los espacios de variabilidad en fonología. Voz y Letra. Revista de Filología, 2(1), 17-38.

Caravedo, R. (2014). Percepción y variación lingüística. Enfoque sociocognitivo. Madrid/Frankfurt: Iberoamericana/Vervuert.

Cuba, M. (1996). El castellano hablado en Chincha. Lima: Escuela de Postgrado UNMSM.

DeMello, G. (1993). -ra Vs. -se subjunctive: a new look at an old topic. Hispania, 76(2), 235-244.

Fleishman, S. (1989). A basic linguistic metaphor. Studies in language, 13(1), 1-50.

Labov, W. (1978). Where does the linguistic variable stop? A response to Beatriz Lavandera. Working papers in Sociolinguistics, 44, 6-23.

Labov, W. (1983 [1972]). Modelos sociolingüísticos. Madrid: Cátedra.

Lamíquiz, V. (1971). Cantara y cantase. Revista de filología española, 54, 1-11. Recuperado de http://xn--revistadefilologiaespaola-uoc.revistas.csic.es/index. $\mathrm{php} / \mathrm{rfe} / \mathrm{article} / \mathrm{view} / 864 / 1271$.

Lavandera, B. (1984 [1978 y 1979]). Variación y significado. Buenos Aires: Hachette.

Navarro, M. (1990). La alternancia -ra/-se y -ra/-ría en el habla de Valencia (Venezuela). Thesaurus, 45(2), 481-488. Recuperado de http://cvc.cervantes. es/lengua/thesaurus/pdf/45/th_45_002_177_o.pdf

Real Academia Española. (2009). Nueva gramática de la lengua española. Morfología y Sintaxis. Madrid: Espasa.

Samamé, A. (2018). ¿Cantara o cantase?: un estudio sociolingüístico del imperfecto y pluscuamperfecto del subjuntivo en hablantes limeños con grado de instrucción superior (Tesis para optar el grado académico de Magíster en Lingüística). Pontificia Universidad Católica del Perú, Lima.

Serrano, M. (1996). El subjuntivo -ra y -se en oraciones condicionales. Estudios filológicos, 31, 129-140.

Williams, A. (1982). The use of -ra and -se Forms of the past subjunctive in Navarre. Hispania, 65(1), 89-93.

Wrigth, L. (1926). The subjunctive forms in -ra and -se in Spanish-American Speech. Hispania, 9(3), 170-173. 